EPJ Web of Conferences 41, 09005 (2013)

DOI: $10.1051 /$ epjconf/20134109005

(C) Owned by the authors, published by EDP Sciences, 2013

\title{
Metamaterial-Enhanced Nonlinear Terahertz Spectroscopy
}

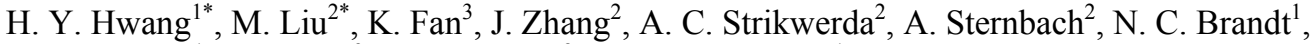 \\ B. G. Perkins ${ }^{1}$, X. Zhang ${ }^{3}$, R. D. Averitt ${ }^{2}$, and K. A. Nelson ${ }^{1}$ \\ ${ }^{1}$ Massachusetts Institute of Technology, Department of Chemistry, Cambridge, MA 02139 USA \\ ${ }^{2}$ Boston University, Department of Physics, Boston, MA 02215 USA \\ ${ }^{3}$ Boston University, Department of Mechanical Engineering, Boston, MA 02215 USA \\ ${ }^{*}$ Contributed equally to this work
}

\begin{abstract}
We demonstrate large nonlinear terahertz responses in the gaps of metamaterial split ring resonators in several materials and use nonlinear $\mathrm{THz}$ transmission and THz-pump/THz-probe spectroscopy to study the nonlinear responses and dynamics. We use the field enhancement in the SRR gaps to initiate high-field phenomena at lower incident fields. In vanadium dioxide, we drive the insulator-tometal phase transition with high-field $\mathrm{THz}$ radiation. The film conductivity increases by over two orders of magnitude and the phase transition occurs on a several picosecond timescale. In gallium arsenide, we observe high-field transport phenomena, including mobility saturation and impact ionization. The carrier density increases by up to ten orders of magnitude at high fields. At the highest fields, we demonstrate THz-induced damage in both vanadium dioxide and gallium arsenide.
\end{abstract}

\section{Introduction}

Developments in high-field terahertz $(\mathrm{THz})$ pulse generation have recently led to the observation of nonlinear $\mathrm{THz}$ phenomena in a several systems ranging from electronic effects in semiconductors to vibrational nonlinearities in molecular crystals [1-6]. However, there still exist many limitations in the kinds of nonlinearities observed due to limitations in peak electric field strengths in different parts of the THz spectrum. In this work, we present a platform for nonlinear THz spectroscopy based on metamaterial split ring resonators (SRRs). We use the inherent field-strength enhancement in the capacitive gaps of gold SRRs to initiate large nonlinear responses in a variety of materials. These effects include a THz-induced insulator-to-metal phase transition (IMT) in vanadium dioxide $\left(\mathrm{VO}_{2}\right)$, and impact ionization in bulk gallium arsenide (GaAs). The $\mathrm{THz}$ field enhancement due to the metamaterials changes the properties of the material in the capacitive gaps leading to a global change in the metamaterial response. The metamaterial structures enhance both the local $\mathrm{THz}$ field strength and the global $\mathrm{THz}$ probe sensitivity to the localized response. We use nonlinear $\mathrm{THz}$ transmission and THz-pump/THz-probe experiments to study strong nonlinear responses and their picosecond dynamics.

This is an Open Access article distributed under the terms of the Creative Commons Attribution License 2.0, which permits unrestricted use, distribution, and reproduction in any medium, provided the original work is properly cited. 


\section{Results and Discussion}

Figure 1a shows THz electric field enhancement in the gaps of a gold SRR. The field is enhanced by a factor of 20-30 over a broad range of frequencies (fig 1c), keeping the THz pulse relatively short in duration in the SRR gaps (fig 1b). The broadband enhancement is due to the close proximity in frequency of the dipole antenna and LC circuit resonances of the SRR. Upon intense THz excitation, the enhanced $\mathrm{THz}$ field induces a large change in the conductivity of $\mathrm{VO}_{2}$, shorting the SRR gaps, as evidenced by the suppression of the $0.4 \mathrm{THz}$ resonance at high in-gap field strengths and the shift in the resonant frequency of the SRR from about $0.4 \mathrm{THz}$ at low in-gap fields to $0.8 \mathrm{THz}$ at large ingap fields (fig 1d). Full-wave simulations of the SRR response as a function of in-gap conductivity (fig 1e) show that the conductivity of the $\mathrm{VO}_{2}$ film changes by over two orders of magnitude. Further dynamic THz-pump/THz-probe studies of the response show that this conductivity change persists for longer than 100 picoseconds.
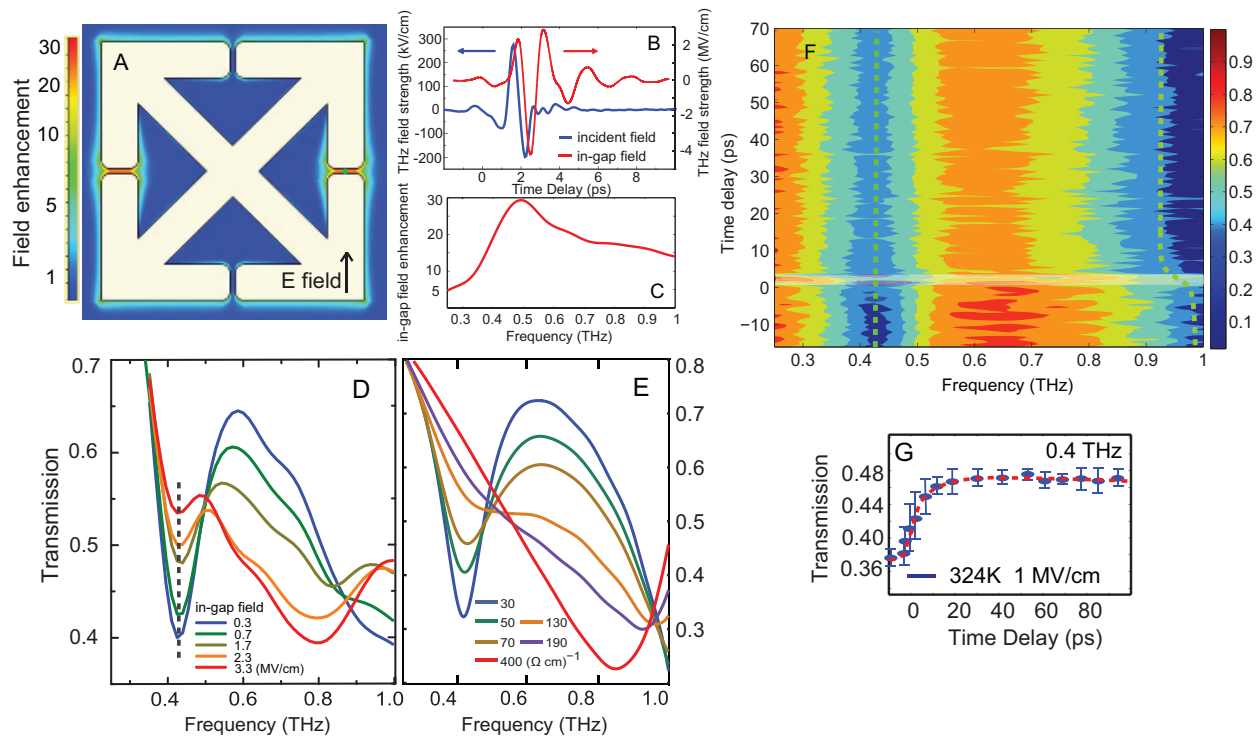

Fig. 1. Nonlinear $\mathrm{THz}$ responses of metamaterials on vanadium dioxide. (A) Calculated $\mathrm{THz}$ field enhancement as a function of position at the time delay $(2.3 \mathrm{ps})$ where the maximum field enhancement occurs in the horizontally oriented gaps. The gold SRRs were $76 \mu \mathrm{m} \times 76 \mu \mathrm{m}$ with a periodicity of $100 \mu \mathrm{m}$ and they were deposited on a $\mathrm{VO}_{2}$ layer. (B) Simulated time-dependent $\mathrm{THz}$ field (red) in the horizontal gaps utilizing experimental data (blue) as the input incident field. (C) Frequency-dependent in-gap field enhancement obtained from the ratio of Fourier amplitudes of the simulated in-gap and measured incident fields in (B). (D) Experimental data showing field-dependent nonlinear $\mathrm{THz}$ transmission spectra of $\mathrm{SRRs}$ on $\mathrm{VO}_{2}$ at $324 \mathrm{~K}$. As the field strength is increased, the transmission at the resonant frequency increases and the resonance nearly disappears. The dipole resonance frequency redshifts to below $0.8 \mathrm{THz}$. (E) Full-wave simulations of THz SRR responses with different in-gap conductivities (assuming the conductivity of $\mathrm{VO}_{2}$ only changes in the SRR gaps). (F) Frequency-resolved THz-pump/THz-probe data obtained through Fourier transformation of transmitted probe time-dependent field profiles. The transmission is plotted versus frequency and pump-probe delay. (G) The transmission at $0.4 \mathrm{THz}$ as a function of pump-probe delay corresponding to an in-gap $\mathrm{THz}$ pump field strength of $1 \mathrm{MV} / \mathrm{cm}$ at $324 \mathrm{~K}$.

Similar measurements and calculations have demonstrated carrier generation in GaAs. Figure 2a shows experimental nonlinear $\mathrm{THz}$ transmission under different incident field strengths. As the conductivity in the gap increases, the SRR resonance at $0.85 \mathrm{THz}$ grows weaker and shifts to the blue. Simulations indicate a change in the carrier density of up to ten orders of magnitude where the conductivity increases from $1 \times 10^{-7}(\Omega \cdot \mathrm{cm})^{-1}$ to $20(\Omega \cdot \mathrm{cm})^{-1}$ and the mobility decreases by an order of 
magnitude. The change in conductivity is likely due to carrier generation by impact ionization through which conduction electrons that have been driven to multi-eV energies by the $\mathrm{THz}$ pulse liberate valence electrons to create additional carriers.
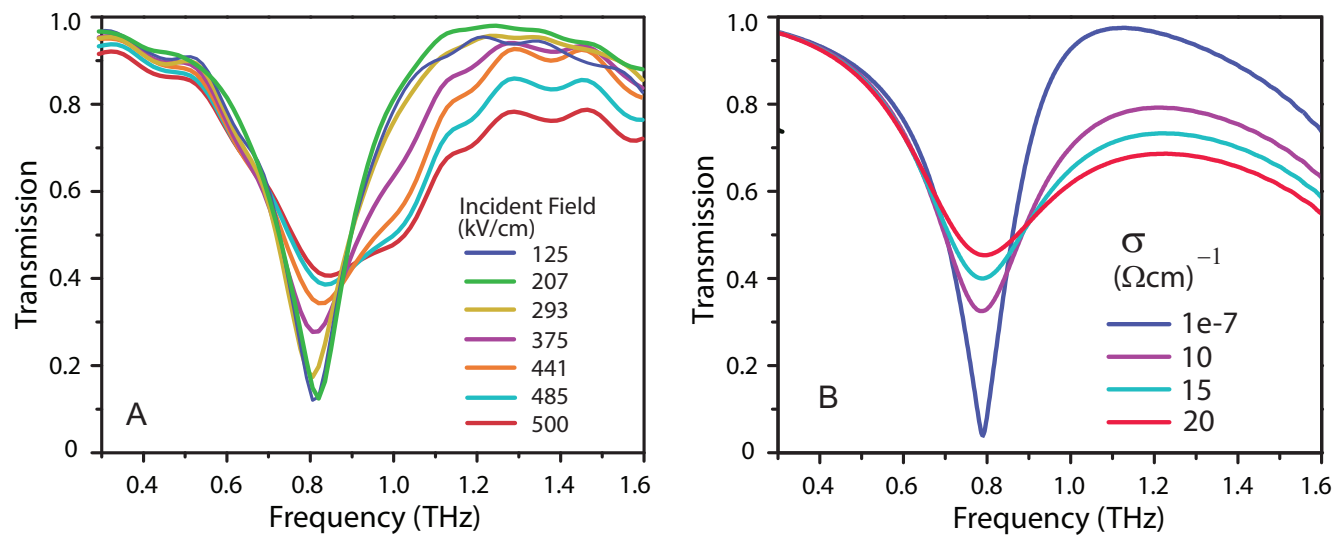

Fig. 2. Nonlinear $\mathrm{THz}$ responses of metamaterials on gallium arsenide. (A) Experimental data showing fielddependent nonlinear THz transmission spectra of SRRs on GaAs at room temperature. The LC resonance of the SRR is $0.85 \mathrm{THz}$. As the conductivity in the gap increases, the resonance grows weaker. (B) Full wave simulations of the THz SRR response with different in-gap conductivities. The conductivity changes over several orders of magnitude due to carrier generation.

\section{Conclusions}

We have demonstrated a powerful and general platform for studying highly nonlinear THz-induced responses from metamaterial-enhanced fields in the gaps of SRRs. In $\mathrm{VO}_{2}$, we studied the THzinduced IMT, which occurs on a picosecond timescale and persists for at least several hundred picoseconds. In GaAs, we studied carrier generation by impact ionization yielding changes of in-gap conductivity of several orders of magnitude. This approach has potential applications in $\mathrm{THz}$ detection and imaging.

Acknowledgements. The authors acknowledge support from DOE-BES under grant DE-FG0209ER46643 and from ONR grant N00014-09-1-1103.

\section{References}

1. K.-L. Yeh, M. C. Hoffmann, J. Hebling, and K. A. Nelson, Appl. Phys. Lett. 90, 171121 (2007)

2. H. Hirori, A. Doi, F. Blanchard, and K. Tanaka, Appl. Phys. Lett. 98, 091106 (2011)

3. M. C. Hoffmann, J. Hebling, H. Y. Hwang, K.-L. Yeh, and K. A. Nelson, Phys. Rev. B 79, 161201(R) (2009)

4. H. Hirori, K. Shinokita, M. Shirai, S. Tani, Y. Kadoya, and K. Tanaka, Nature Communications 2, 594 (2011)

5. J. Hebling, M. C. Hoffmann, H. Y. Hwang, K.-L. Yeh, and K. A. Nelson, Phys. Rev. B 81, $035201(2010)$

6. M. Jewariya, M. Nagai, and K. Tanaka, Phys. Rev. Lett. 105, 203003 (2010) 